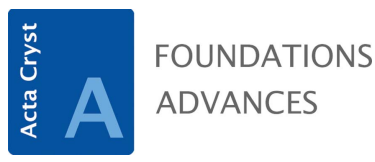

ISSN 2053-2733
Keywords: Aperiodic 2018; aperiodic crystals; quasicrystals; incommensurate composites; incommensurate modulated phases; intergrowths; proteins.

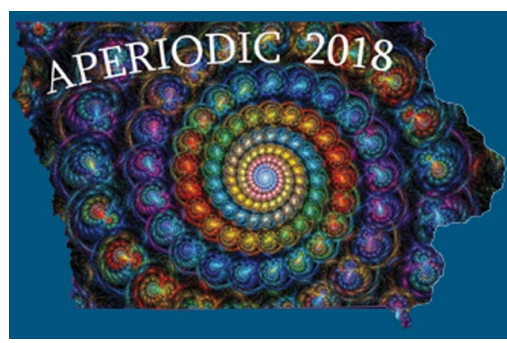

(C) 2019 International Union of Crystallography

\section{Aperiodic order coming of age: from inorganic materials to dynamic protein superstructures}

\author{
Gloria Borgstahl, ${ }^{a}$ Alan I. Goldman ${ }^{\mathrm{b}}$ and Patricia A. Thiel ${ }^{\mathrm{C} *}$
}

aUniversity of Nebraska Medical Center, The Eppley Institute for Research in Cancer and Allied Diseases, Fred \& Pamela Buffett Cancer Center, 986805 Nebraska Medical Center, Omaha, NE 68198-6805, USA, b lowa State University, Department of Physics and Astronomy, 12 Physics Hall, Ames, IA 500-1021, USA, and 'lowa State University, Department of Chemistry, 1605 Gilman Hall, 2415 Osborn Drive, Ames, IA 50011-1021, USA. *Correspondence e-mail: pthiel@ameslab.gov

Aperiodic crystals, once regarded as anomalies or outliers in the field of crystallography, have taken center stage since Dan Shechtman's discovery of icosahedral quasicrystals in an Al-Mn alloy in 1982. The subsequent publication of the now-famous Physical Review Letter, Metallic phase with long-range orientational order and no translational symmetry, with Ilan Blech, Denis Gratias and John Cahn in 1984 ignited a firestorm of controversy centered on the proper definition of crystalline materials. Ultimately, quasicrystals and, more generally, aperiodic crystals were 'validated' and assumed their place among the vast array of crystalline forms when the definition of a crystal was modified to read as follows:

A material is a crystal if it has essentially a sharp diffraction pattern.

The word 'essentially' means that most of the intensity of the diffraction is concentrated in relatively sharp Bragg peaks, besides the always-present diffuse scattering. In all cases, the positions of the diffraction peaks can be expressed by

$$
\mathbf{H}=\sum_{i=1}^{n} h_{i} \mathbf{a}_{i}^{*} \quad(n \geq 3) .
$$

Here $\mathbf{a}_{i}^{*}$ and $h_{i}$ are the basis vectors of the reciprocal lattice and integer coefficients, respectively, and the number $n$ is the minimum for which the positions of the peaks can be described with integer coefficient $h_{i}$. The conventional crystals are a special class, though very large, for which $n=3$ (see the IUCr Online Dictionary of Crystallography entry on 'Crystal' at http://reference.iucr.org/dictionary/crystal).

Aperiodic crystals are ubiquitous in Nature and in the laboratory, and they have been acknowledged, studied and puzzled over since the earliest days of the science of crystallography, pre-dating even the use of X-rays to study atomic scale structure (Pina \& López-Acevedo, 2016). They also come in several flavors, such as incommensurately modulated phases associated with atomic displacements or compositional modulations, incommensurate composites or intergrowth compounds consisting of two or more subsystems and, of course, quasicrystals (Janssen et al., 2018).

Aperiodic crystals are not restricted to bulk inorganic compounds or minerals, as the 9th Conference on Aperiodic Crystals (Aperiodic 2018), held 8-13 July 2018 in Ames, Iowa, demonstrated. Sessions in the programme of this meeting featured Soft Matter Quasicrystals, Surfaces and Overlayers, and Aperiodic Biological Systems, and also focused on the properties of aperiodic and complex systems including Growth and Stability, Electrical, Magnetic and Thermal Properties, and Superconductivity.

Aperiodic 2018 was the latest in a series of conferences sponsored by the Commission on Aperiodic Crystals of the International Union of Crystallography (IUCr). These conferences are held every three years, with the most recent meetings in Prague (Czech Republic, 2015), Cairns (Australia, 2012), Liverpool (UK, 2009), Zao (Japan, 2006), Belo Horizonte (Brazil, 2003) and Nijmegen (The Netherlands, 2000). Aperiodic 2018 was the first of this series held in North America.

Among the exciting research presented at Aperiodic 2018, some of the best was presented by students and young investigators. Four formal awards were given in recognition of these excellent studies, two of which are described in this special issue. For 
instance, Nayuta Takemori of Oyakama University described the exciting first observation of superconductivity in a quasiperiodic intermetallic, Al-Zn-Mg. Her careful comparison with approximants in this family revealed that $T_{\mathrm{c}}$ is much lower in the quasicrystal, an effect that she related to the well known pseudogap of the quasicrystal. Ashwin Shahani of the University of Michigan described beautiful X-ray tomography experiments, in which growth and dissolution of the decagonal quasicrystal Al-Co-Ni (from the liquid phase) was observed as a function of time (Han et al., 2019). Through quantitative evaluation, he and his co-workers concluded that growth of the solid proceeds via attachment of large clusters of atoms from the liquid, rather than by attachment of individual atoms. This is relevant to a longstanding debate over the physical versus conceptual importance of clusters in stabilizing quasicrystals and other intermetallics (Henley et al., 2006; Steurer, 2006). Also relevant to this debate was the presentation by Shuang Zhang of Dalian Institute of Technology, which described the most recent advances in using a clusterplus-glue-atom approach for predicting and explaining a wide variety of metallic phases, from metallic glasses to quasiperiodic systems. Paul Klar of Universidad del Pais Vasco (UPV/EHU) tackled the complex, and long-mysterious, mineral known as mullite. This aluminium silicate exhibits both commensurate and incommensurate vacancy-modulated structures as a function of composition. By interweaving density-functional theory (DFT) with superspace refinement of synchrotron single-crystal diffraction data, Klar and coworkers produced models that explain both types of phases over a broad composition range and set an example of how to approach analogous systems (Klar et al., 2019). These four presentations by students and young investigators illustrate exciting directions in aperiodic research, but many others were also represented at the conference and in the Proceedings. In this special issue, we also feature work by Oğuz et al., Paßens \& Karthäuser, Scheid et al., Schenk et al. and Vinokur et al. The interested reader can find all of the conference abstracts in an online archive at Iowa State University (https://lib.dr. iastate.edu/aperiodic2018/2018/).
All modern scientific advances are built upon the shoulders of giants. At Aperiodic 2018 we not only looked forward to new directions, but we also paid tribute to two persons who played important enabling roles in aperiodic research: Ted Janssen, one of the founding fathers of superspace crystallography, and Esther Belin-Ferré, discoverer of the pseudogap in quasicrystals. Both of them provided us with powerful tools and concepts for understanding aperiodic systems; Ted Janssen's contributions to the field are reviewed here by Marc de Boissieu (2019). We look forward to Aperiodic 2021 in Sapporo, Japan, to see what new exciting discoveries await us.

Finally we wish to thank all of our financial sponsors who made this meeting possible, including Iowa State University College of Liberal Arts and Sciences and the Departments of Physics and Astronomy, Materials Science and Engineering, and Chemistry, the IUCr, European C-MAC, and corporate sponsors Bruker, Rigaku, Dectris, Crystals and MiTeGen.

\section{References}

Boissieu, M. de (2019). Acta Cryst. A75, 273-280.

Han, I., Xiao, X., Sun, H. \& Shahani, A. J. (2019). Acta Cryst. A75, 281-296.

Henley, C. L., de Boissieu, M. \& Steurer, W. (2006). Philos. Mag. 86, 1131-1151.

Janssen, T., Chapuis, G. \& de Boissieu, M. (2018). Aperiodic Crystals. From Modulated Phases to Quasicrystals: Structure and Properties, 2nd ed. Chester, Oxford: IUCr/Oxford University Press.

Klar, P. B., Etxebarria, I. \& Madariaga, G. (2019). Acta Cryst. A75, 260-272.

Oğuz, E. C., Socolar, J. E. S., Steinhardt, P. J. \& Torquato, S. (2019). Acta Cryst. A75, 3-13.

Paßens, M. \& Karthäuser, S. (2019). Acta Cryst. A75, 41-49.

Pina, C. M. \& López-Acevedo, V. (2016). Crystals, 6, 137-152.

Scheid, P., Chatelier, C., Ledieu, J., Fournée, V. \& Gaudry, É. (2019). Acta Cryst. A75, 314-324.

Schenk, S., Zollner, E. M., Krahn, O., Schreck, B., Hammer, R., Förster, S. \& Widdra, W. (2019). Acta Cryst. A75, 307-313.

Shechtman, D. S., Blech, I., Gratias, D. \& Cahn, J. W. (1984). Phys. Rev. Lett. 53, 1951-1953.

Steurer, W. (2006). Philos. Mag. 86, 1105-1113.

Vinokur, A. I., Hilleke, K. P. \& Fredrickson, D. C. (2019). Acta Cryst. A75, 297-306. 